\title{
Microscope - A space mission to test the equivalence principle
}

\author{
Meike List ${ }^{1}$, Hanns Selig ${ }^{1}$, Stefanie Bremer $^{1}$ and Claus Lämmerzahl ${ }^{1}$ \\ ${ }^{1}$ ZARM - Center of Applied Space Technology and Microgravity, \\ University of Bremen, \\ Am Fallturm, D-28359 Bremen, Germany \\ email: list@zarm.uni-bremen.de, selig@zarm.uni-bremen.de, \\ bremer@zarm.uni-bremen.de, laemmerzahl@zarm.uni-bremen.de
}

\begin{abstract}
MICROSCOPE is a ESA/CNES space mission for testing the validity of the weak equivalence principle. The mission's goal is to determine the Eötvös parameter $\eta$ with an accuracy of $10^{-15}$. The French space agency CNES is responsible for designing the satellite which is developed and produced within the Myriade series. The satellite's payload T-SAGE (Twin Space Accelerometer for Gravitation Experimentation) consists of two high-precision capacitive differential accelerometers and is developed and built by the French institute ONERA.

As a member of the MICROSCOPE performance team, the German department ZARM performs free fall tests of the MICROSCOPE differential accelerometers at the Bremen drop tower. The project's concepts and current results of the free fall tests are shortly presented.
\end{abstract}

Keywords. Relativity, Gravitation

\section{Overview}

MICROSCOPE is a ESA/CNES $\mu$-satellite project which aims at a high precision test of the Weak Equivalence Principle (WEP) which may be violated as predicted by many quantum theories of gravity (Isham 1996, Haugan \& Lämmerzahl 2000) as well as within the theoretical framework of string theories (Damour \& Polyakov 1994). Thus experiments which test this fundamental principle of general relativity are of big interest, especially concerning the unification of current fundamental theories (quantum chromodynamics, quantum electrodynamics, electroweak theory, general relativity) (e.g. Damour 1996).

The validity of the WEP predicts an equal gravitational acceleration acting on every point mass independent of its composition resulting in identical trajectories in gravitational fields (effects due to the point masses' own gravitational field are neglected). So far this so called universality of free fall is confirmed up to an accuracy of $10^{-13}$ in terms of the Eötvös parameter $\eta$ defined by

$$
\eta=\frac{\mu_{1}-\mu_{2}}{\frac{1}{2}\left(\mu_{1}+\mu_{2}\right)}, \quad \text { with } \quad \mu_{a}=\left(\frac{m_{g}}{m_{i}}\right)_{\text {mass a }}
$$

where $m_{g}$ is the gravitational and $m_{i}$ the inertial mass, respectively. If the WEP holds, the $\eta=0$. With MICROSCOPE $\eta$ will be measured with an accuracy of $10^{-15}$.

Up to now the best WEP tests have been carried out with the help of torsion balance experiments (Su et al. 1994, Smith et al. 1999, Boynton 2000, Schlamminger et al. 2008) which are strongly influenced by tidal effects as well as effects due to the inhomogenity of the Earth' gravitational field. Thus, there is great effort to put free fall experiments into space (e.g. MICROSCOPE, STEP) (Touboul \& Rodrigues 2001, Lockerbie et al. 2001, Worden 1993). 
Since 2001 ZARM cooperates with CNES and ONERA on the ESA/CNES mission MICROSCOPE. ZARM is in charge of, among other tests, testing and verifying the MICROSCOPE payload in free fall at the Bremen drop tower. These tests are essential for the successful development of the T-SAGE instrument. The behaviour of the instrument can only be tested in free fall with very low disturbance levels. Thus, parabolic flights which reach milli-g conditions only, are far away from the requirements that are given by the limited dynamic range of the instrument. Secondly the development of an end-toend simulation of the satellite's and payload's dynamics in space is underway. For these purposes several simulation tools are under construction. The simulations will cover the behavior of the differential accelerometer in space as well as the satellite's interaction with the environment (residual atmosphere, solar wind, radiation pressure, etc.). Finally, the mission data analysis will be carried out at ZARM in cooperation with the French project partners.

\section{Mission design}

MICROSCOPE will be launched in 2012, it is supposed to orbit around the Earth with an altitude of about $800 \mathrm{~km}$. The orbit's eccentricity is planned to be $\epsilon=5 \cdot 10^{-3}$, the satellite's spinning rate is given by two spinning modes which are stabilised by using a star tracker. MICROSCOPE will be a drag-free mission, the control of $\mu N$-thruster application is realised with the help of the common mode signal while scientific data is taken of the differential mode signal.

The science signal will be measured using capacitive sensors. Each sensor unit consists of a slightly modified cylindric test mass (see Figure 1(left)). The motion of the test masses can be detected and stabilised capacitively along six degrees of freedom. Electrostatic forces will compensate disturbances due to environmental and coupling effects, thus ensuring freely falling test masses. Environmental influences which may affect the test masses geodetic motion are e.g. the Earth' atmosphere and albedo, solar pressure, etc.

\section{Free fall tests}

Since a residual acceleration of $5 \cdot 10^{-7} \mathrm{~m} / \mathrm{s}^{2}$ during a drop at the Bremen drop tower is the default value defined by ONERA, disturbances acting on the test masses had to be scaled down using a free-flyer platform. Operation of this platform consists of its disengagement, of damping of vibrations which are a result of the natural oscillation of the system, as well as construction and manufacturing of an appliance for determining the
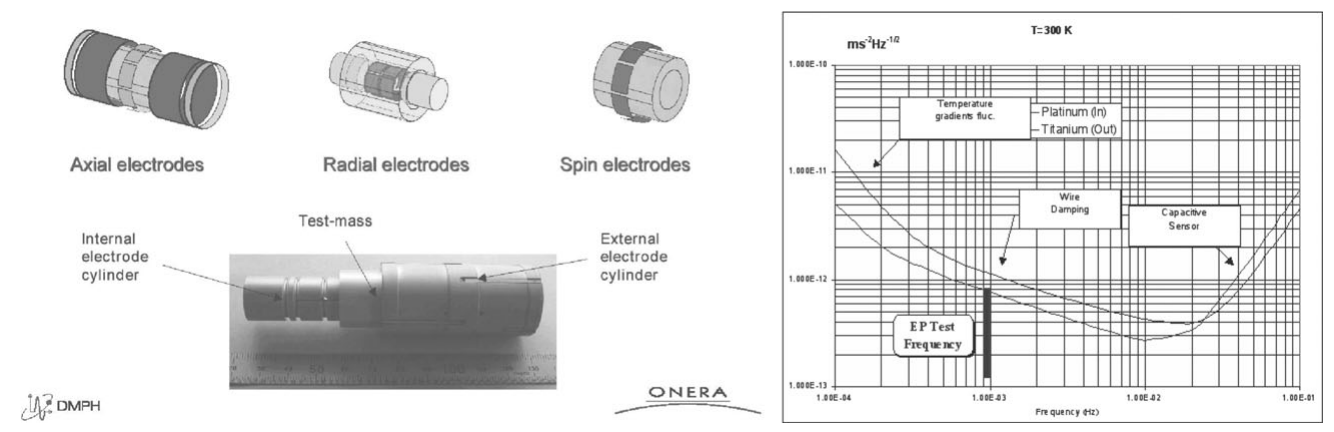

Figure 1. Sensor unit (left) as well as the performance (right) of T-SAGE 

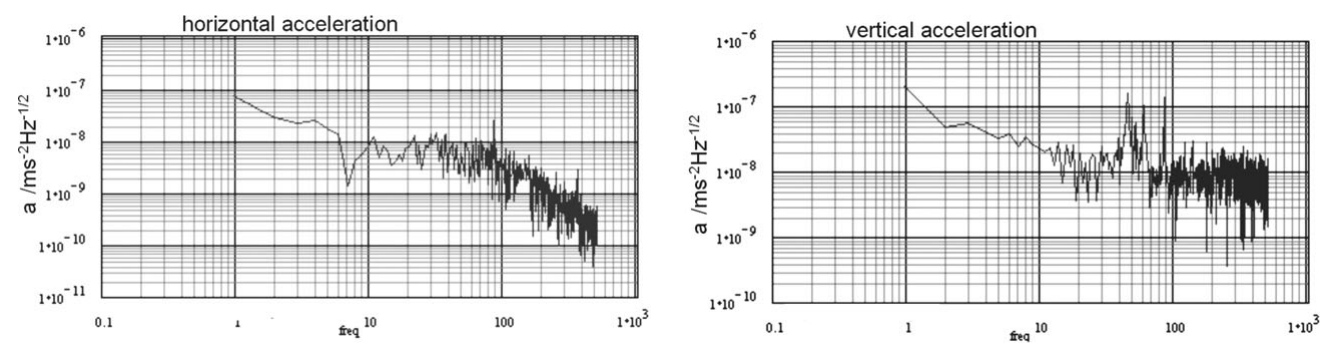

Figure 2. Residual accelerations in horizontal (left) and vertical (right) direction during drop

center of gravity of free-flyer and test masses within an accuracy of $1 \mathrm{~mm}$. For these purposes more than 50 drops were performed and led finally to a successful implementation of a $\mu \mathrm{g}$-laboratory.

A standard drop using the free-flyer is characterised by:

- $\mu \mathrm{g}$-duration: $2-3 \mathrm{~s}$,

- $\mu \mathrm{g}$-level: $10^{-7} \mathrm{gHz}^{-1 / 2}-10^{-8} \mathrm{gHz}^{-1 / 2}$ in horizontal and vertical direction (see Figure 2).

The drop tower test campaigns for the MICROSCOPE instrument will contain 60 drops in 2009 and 2010 for tests and verification of EM and QM hardware. The campaign includes checking of sensor characteristics and performance, verification of the electronic conrol loop behaviour as well as tests of data acquisition and control procedures.

\section{Acknowledgement}

The authors like to thank the DLR Space Agency (Deutsches Zentrum für Luft- und Raumfahrt) for funding the project. Additionally we would like to thank P. Touboul, M. Rodrigues, B. Foulon, R. Chhun, V. Josselin, F. Liorzou (ONERA) and G. Metris (OCA) for fruitful discussions. This work is supported by DLR with funds of the BMWi (FKZ 50 OY 0801).

\section{References}

Isham, C. J. 1996, Class. Quantum Grav., 13, A5

Haugan, M. P. \& Lämmerzahl, C. 2001, Principles of equivalence: their role in gravitation physics and experiments that test them, Gyros, Clocks, Interferometers, ...: Testing Relativistic Gravity in Space (Lecture notes in Physics), Springer Verlag

Damour, T. \& Polyakov, A. M. 1994, Nucl.Phys., 423, 532

Damour, T. 1996, Class. Quantum Grav., 13, A33

Touboul, P. \& Rodrigues, M. 2001, Class. Quantum Grav., 18, 2487

Lockerbie, N. A., Mester, J. C., Tori, R., Vitale, S., \& Worden, P. W. 2001, STEP: A Status Report, Gyros, Clocks, Interferometers, ...: Testing Relativistic Gravity in Space (Lecture notes in Physics), Springer Verlag

Worden, P. W. 1993, STEP Symposium, Pisa, ESA Publications Division

Su, Y., Heckel, B. R., Adelberger, E. G., Grundlach, J. H., Harns, M., Smith, G. L., \& Swanson, H. E. 1994, Phys. Rev. D, 50, 3614

Smith, C. L., Hoyle, C. D., Grundlach, J. H., Adelberger, E. G., Heckel, B. R., \& Swanson, H. E. 1999, Phys. Rev. D, 61, 2201

Boynton, P. E. 2000, Class. Quantum Grav., 17, 2319

Schlamminger, S., Choi, K.-Y., Wagner, T. A., Gundlach, J. H., \& Adelberger, E. G. 2008, Phys. Rev. Lett., 100, 041101 